\title{
Correlation of nuclear ploidy with histology in adenomatous polyps of colon
}

\author{
L R JARVIS, P S GRAFF, R WHITEHEAD \\ From the Department of Pathology, Flinders Medical Centre, Bedford Park, South Australia
}

SUMMARY Histological sections of adenomatous polyps of the colon showing carcinoma were studied by video image analysis. Nuclear DNA content and morphology were measured in regions identified as either dysplasia, carcinoma confined to the mucosa, or carcinoma invading the muscularis mucosa. Where carcinoma was present, areas of dysplasia in the same polyp were found to have similar distributions of nuclear DNA content and size, supporting the notion that adenomatous polyp becomes cancer. The method can be used to detect those regions in sections of adenomatous polyps with the most severe nuclear abnormality.

The risk of carcinoma of the colon developing from adenomatous polyps is well recognised, but it is probably low and unpredictable. ${ }^{1}$ There is evidence to suggest that the degree of abnormality, as expressed in epithelial atypia or dysplasia in a given polyp, may reflect the degree of increased risk for the patient of developing subsequent polyps and cancer. ${ }^{2}$ In any one case, however, it is impossible to determine the degree of risk with any accuracy by subjective examination. A more objective means of detecting abnormality is therefore necessary.

It has been shown, using flow cytometry, that adenomatous polyps showing severe atypia, or carcinoma by our definitions, may be aneuploid, ${ }^{3}$ suggesting that ploidy analysis may provide the means of detecting carcinoma, or the potential for its development. This technique, however, entails disaggregation of tissue to produce suspensions of nuclei and may have the effect of diluting any abnormality, especially if this is focally distributed.

The present study was aimed at measuring, by image analysis densitometry, the size and nuclear DNA content of nuclei in histological sections of adenomatous polyps. In this way the nuclear characteristics of focal regions of carcinoma could be studied without contamination by unwanted tissue components. Furthermore, the precise correlation with histology allows the dysplasia present with carcinoma in the same specimen to be studied independently.

Accepted for publication 8 July 1986

\section{Material and methods}

Paraffin embedded specimens of 17 adenomatous polyps were selected, of which seven showed evidence of carcinoma. In two cases the carcinoma was confined to the mucosa. In three cases the carcinoma showed invasion of the muscularis mucosa, and in two the invasive carcinoma comprised the predominant tissue. The remaining 10 cases consisted of tubulovillous adenomas showing varying degrees of dysplasia.

Serial sections of $4 \mu \mathrm{m}$ in thickness were prepared from each specimen. One section was stained with haematoxylin and eosin for subjective examination and the other for DNA measurement using the Feulgen technique. ${ }^{45}$

Regions within the sections were identified as either normal, dysplastic, or carcinoma but still confined within the mucosa, or as invasive carcinoma. Konishi and Morson ${ }^{2}$ suggested that the degree of dysplasia in adenomas, judged by subjective criteria, is in some way related to malignant potential. We therefore selected the most severe areas of dysplasia using their criteria. Carcinoma confined to the mucosa was recognised as an area with features of carcinomathat is, nuclear atypia, loss of mucin secretion, and abnormal gland in gland formation and stromal desmoplasia, which were similar to the appearances in areas of invasive carcinoma (fig 1). ${ }^{6}$ The definition of invasive carcinoma was invasion of muscularis mucosa into submucosa by carcinoma and an associated desmoplastic reaction. Four histological categories were thus defined: invasive carcinoma; carcinoma 


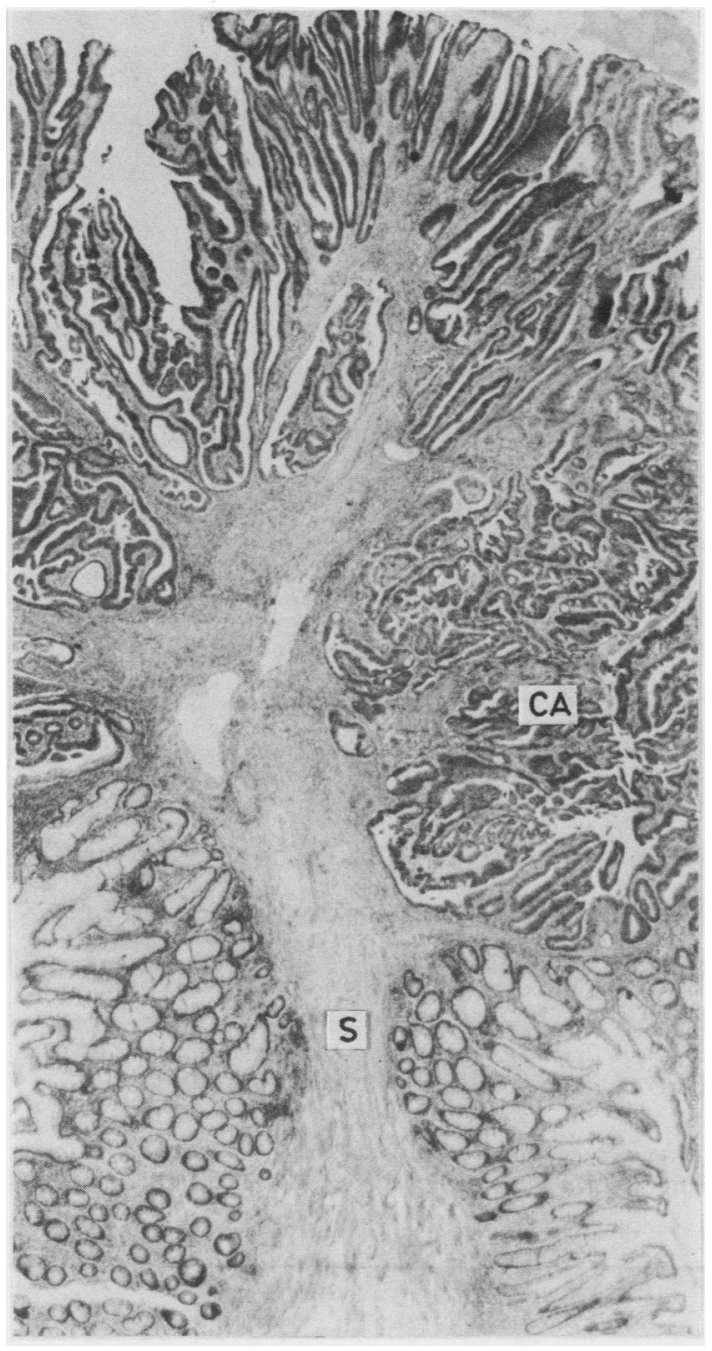

Fig 1 Tubulovillous adenoma with carcinoma confined to mucosa. Note stalk (S) and one of the more conspicuous areas of carcinoma $(C A)$. (Haematoxylin and eosin.) $\times 25$.

confined to the mucosa (fig 2); dysplasia in adenomatous areas in association with carcinoma (fig 3); and dysplasia associated with adenoma alone.

These regions were outlined on the reference slide, and corresponding regions in the Feulgen stained slide were identified for measurement. In total there were 19 regions of dysplasia, three of carcinoma confined to the mucosa, and eight of carcinoma invading the submucosa. Between 200 and 300 epithelial nuclei were measured in each region using a microcomputer image analysis system designed in our laboratory. ${ }^{7}$ Area was expressed in pixel units, with one pixel equivalent to $0.43 \mu \mathrm{m}$ with the $\times 40$ objec- tive, which was used for all measurements. Density measurements were expressed in arbitrary integrated optical density (IOD) units. The system incorporated appropriate corrections for microdensitometric errors. Only those epithelial nuclei with minimal overlap and those which appeared by focusing to be wholly contained in the thickness of the section were selected for measurement. The integrated optical density proportional to the DNA content and profile area was measured for each nucleus. About 50 nuclei of apparently normal morphology were included in the measurements for each case to establish the normal diploid range. A distribution histogram of integrated optical density and scatterplot of nuclear area and integrated density was prepared for each selected region.

\section{Results}

The graphical results indicate a range of increasing nuclear density and size. Partial sectioning or overlap of some of the nuclei contributed to broadening of the ploidy distribution profiles. Nevertheless, histograms could still be classified into four types, as established in our previous study (figs 4-7). ${ }^{8}$ Type 1 is a simple diploid profile with a small proportion of dividing nuclei; type 2 shows a diploid profile with an increased proportion of $4 \mathrm{c}$ nuclei; type 3 shows DNA values in excess of $4 \mathrm{c}$ and with peaks discernible at the $2 c, 4 c$, and $8 \mathrm{c}$ positions; type 4 is an abnormal distribution with peaks at irregular ploidy intervals and a large proportion of nuclei in excess of the $4 \mathrm{c}$ complement. Table 1 shows the histogram types for the 30 regions measured for the 17 cases.

Table 2 indicates the proportions of the histogram types for the four histological categories. Of the eight regions identified as carcinoma with invasion across the muscularis mucosa, two were diploid and six showed abnormally high nuclear DNA values, of which three displayed highly irregular ploidy distributions. In contrast, only one of the three regions of carcinoma confined to the mucosa could be interpreted as showing nuclear DNA content in excess of the normal $4 \mathrm{c}$ level. It seemed to be type 3 with regular ploidy peak intervals (fig 6a). When this type of carcinoma was diploid it could be distinguished from the diploid dysplasias (fig $4 \mathrm{~b}$ ) by the increased nuclear size (fig 8a). The two regions of invasive carcinoma displaying diploid type 2 profiles also showed increased nuclear size and also showed an increased proportion of dividing $(2 n-4 n)$ nuclei (fig $8 b)$.

Four of the nine regions identified as severe dysplasia had distributions of abnormally high nuclear DNA values. In the case of carcinoma confined to the mucosa showing an abnormal ploidy composition (fig 6a) the area of most severe dysplasia in the same 


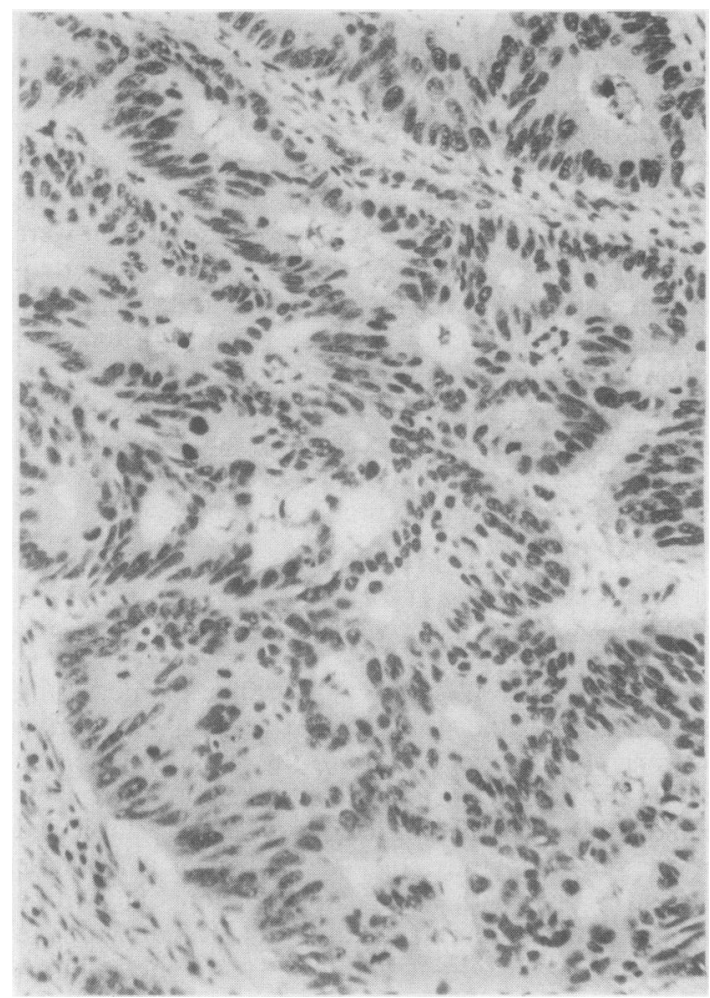

Fig 2

Fig 2 Region of carcinoma confined to mucosa, adjacent to area of severe dysplasia shown in fig 3, displaying nuclear atypia, loss of mucin secretion, and abnormal gland in gland formation. Histogram relating to this area is shown in fig $6 a$.

(Haematoxylin and eosin.) $\times 25$.

Fig 3 Adjacent area of severe dysplasia showing epithelial atypia. Nuclei are large, pleomorphic, and hyperchromatic with mucin secretion present. Histogram relating to this area is shown in fig 5a. (Haematoxylin and eosin.) $\times 25$.

Table 1 Histogram types for 30 regions measured for 17 cases

\begin{tabular}{|c|c|c|c|c|}
\hline Case No & $\begin{array}{l}\text { Carcinoma } \\
\text { invasive }\end{array}$ & $\begin{array}{l}\text { Carcinoma } \\
\text { confined }\end{array}$ & $\begin{array}{l}\text { Severe dysplasia } \\
\text { with carcinoma }\end{array}$ & $\begin{array}{l}\text { Dysplasia } \\
\text { with adenoma }\end{array}$ \\
\hline $\begin{array}{l}1 \\
2 \\
3 \\
4 \\
5 \\
6 \\
7 \\
8-17\end{array}$ & $\begin{array}{l}2,3 \\
3,4 \\
4,4 \\
2 \\
3\end{array}$ & ${ }_{3}^{1,2}$ & $\begin{array}{l}1 \\
3 \\
2,3 \\
3 \\
3 \\
1,2 \\
2\end{array}$ & $1(10)$ \\
\hline
\end{tabular}

Table 2 Proportions of histogram types for four histological categories

\begin{tabular}{lcccc}
\hline & \multicolumn{4}{l}{ Histogram type } \\
\cline { 2 - 5 } & 1 & 2 & 3 & 4 \\
\hline Carcinoma invasive & 0 & 2 & 3 & 3 \\
Carcinoma confined & 1 & 1 & 1 & 0 \\
Dysplasia or carcinoma & 2 & 3 & 4 & 0 \\
Dysplasia or adenoma & 10 & 0 & 0 & 0 \\
\hline
\end{tabular}

lesion (fig 5a), but which by our definitions could not be described as carcinoma, showed similar nuclear DNA and size distributions but with more $2 \mathrm{c}$ nuclei of smaller size (less than 200 pixels area) and a reduced proportion of nuclei with high DNA content (greater than 20 IOD units). This similarity of ploidy abnormality was also found in regions of severe dysplasia in two polyps with associated invasive carcinoma (fig 9b). The 10 adenomas of varying degrees of dysplasia unassociated with carcinoma were all 

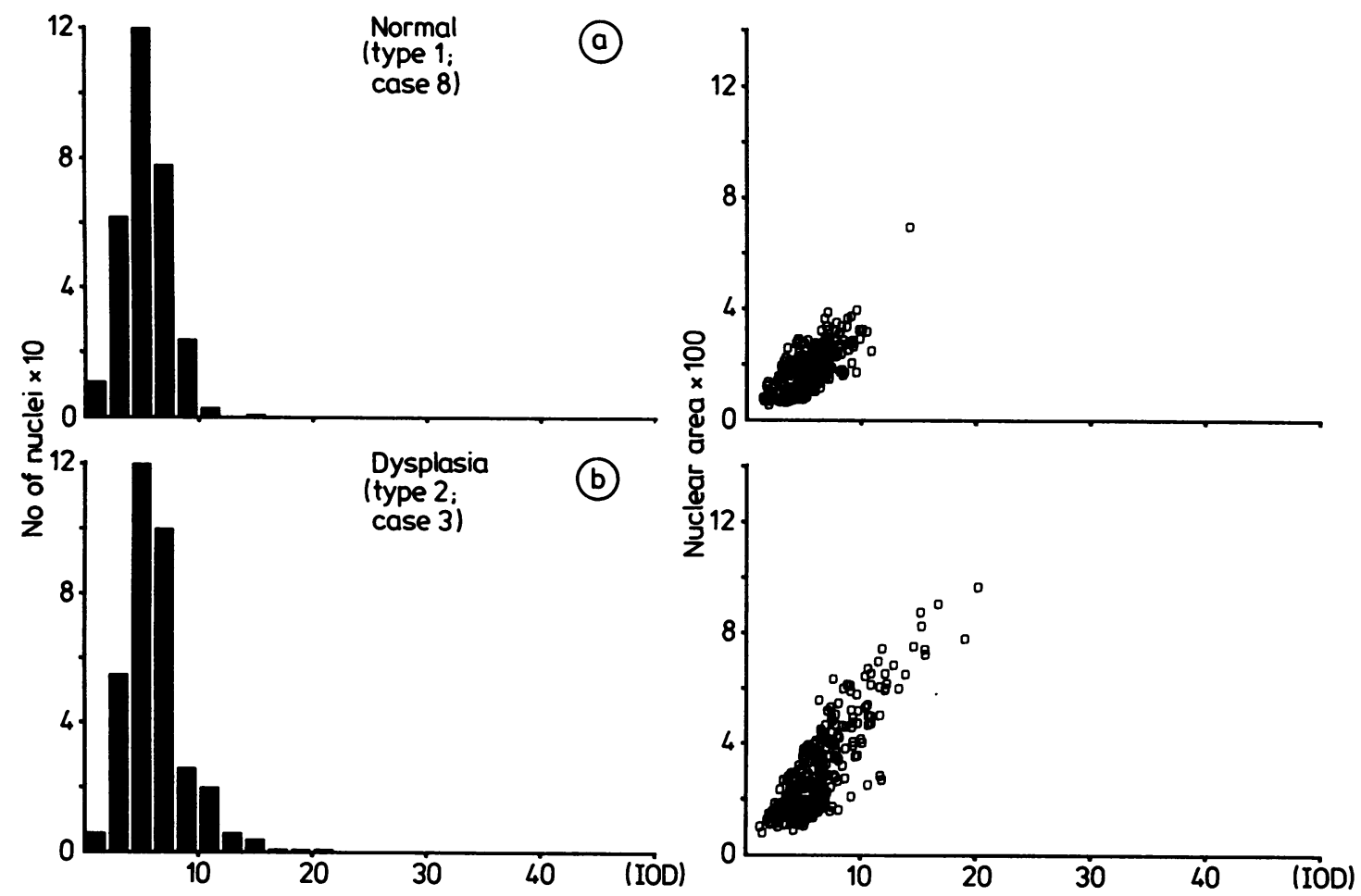

Fig 4
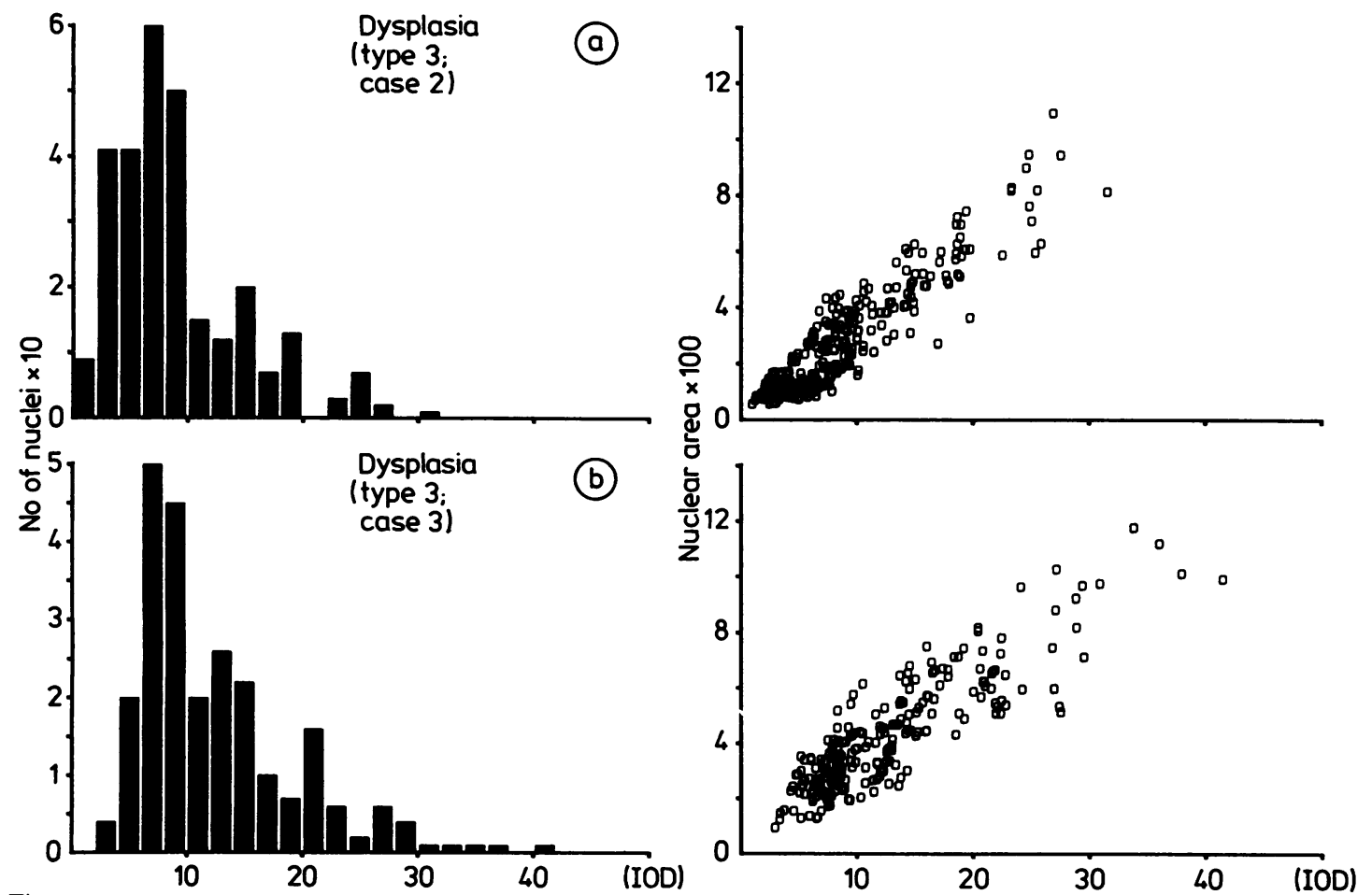

Fig 5

Figs 4-7 Histograms of nuclear DNA content in arbitrary integrated optical density units (IOD) and corresponding scatterplots of integrated density against nuclear profile area (arbitrary units) for selected regions from sectioned adenomatous polyps. Histological definition and histogram type is shown for each region. Increased DNA content and nuclear size are related to degree of histological abnormality. 
Fig 6
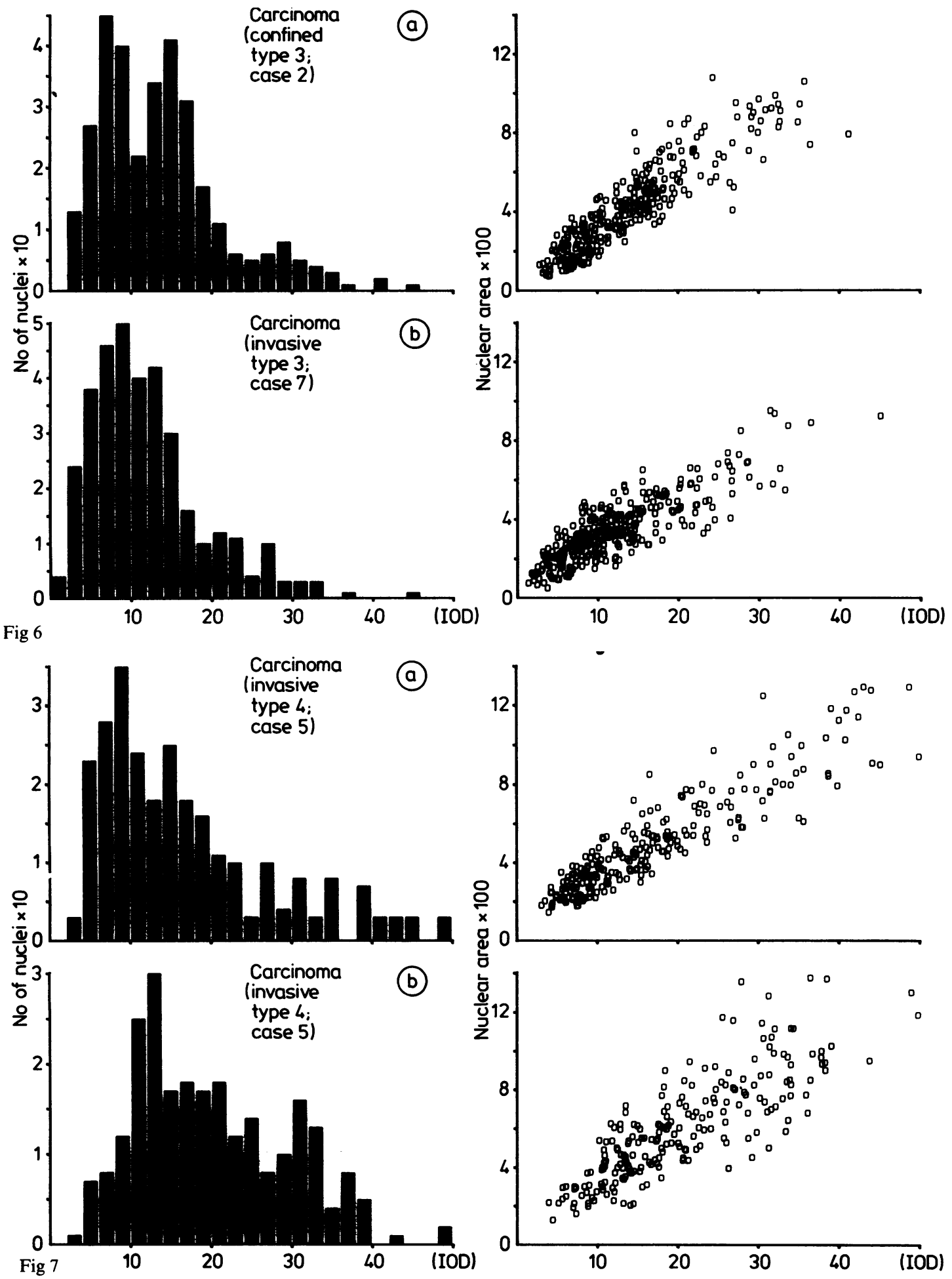

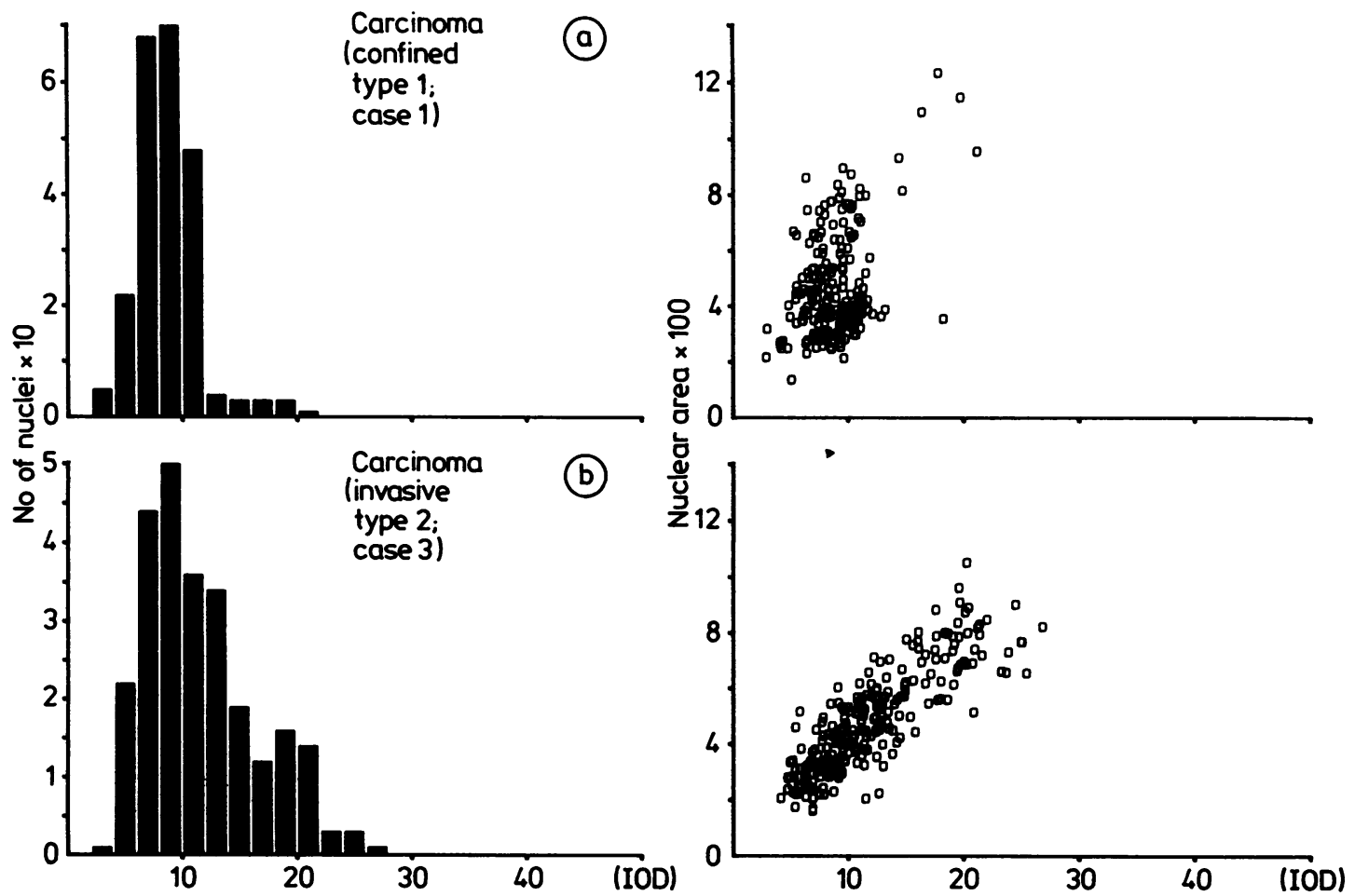

Fig 8 Histograms and scatterplots for carcinoma confined to mucosa (a) and invasive carcinoma (b) showing increased nuclear size compared with dysplasia and increased proportion of $4 n$ nuclei for invasive carcinoma.
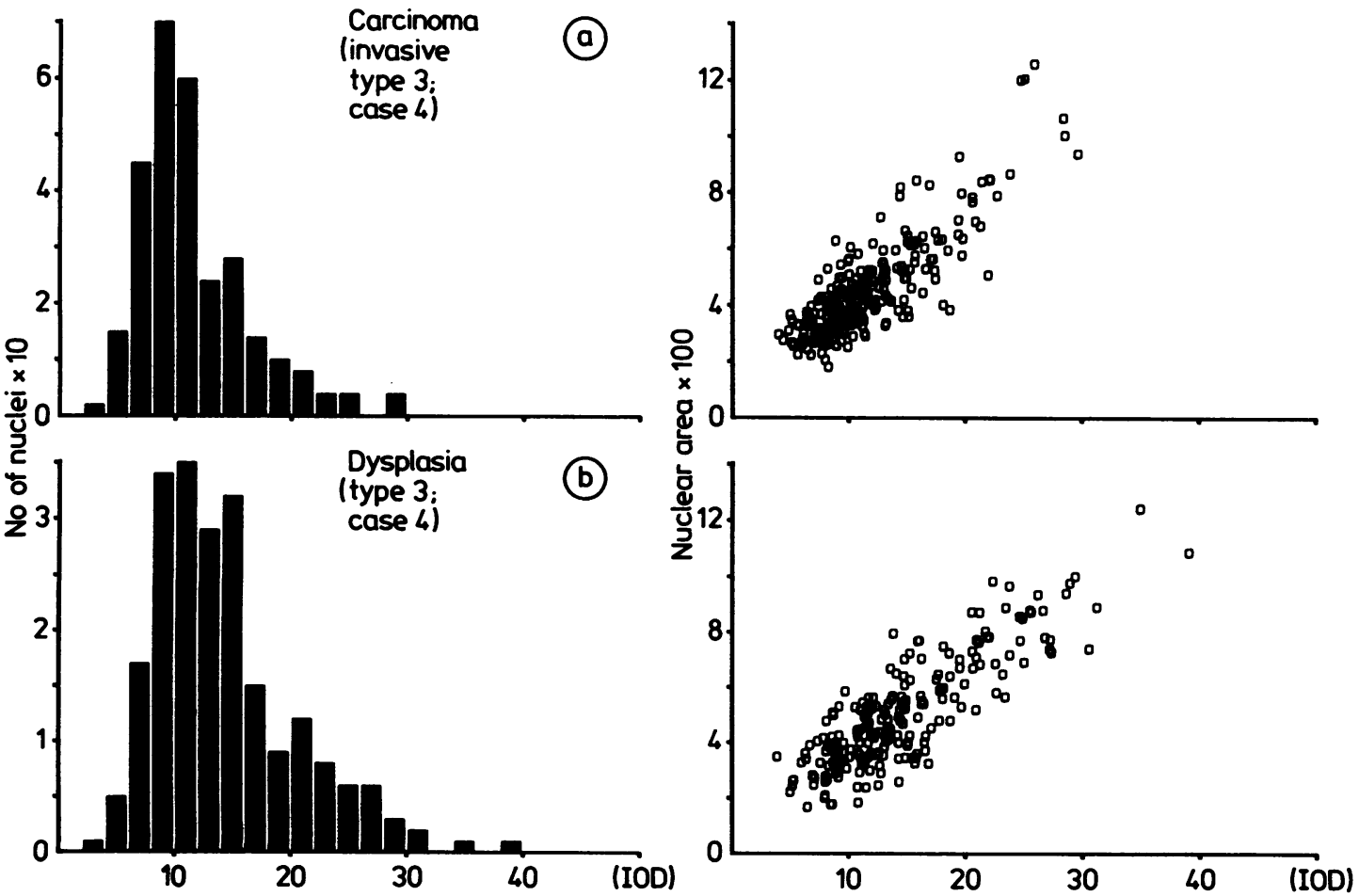

Fig 9 Case with invasive carcinoma showing similarity in distributions of nuclear density and size between invasive region (a) and region of severe dysplasia in same polyp (b). 

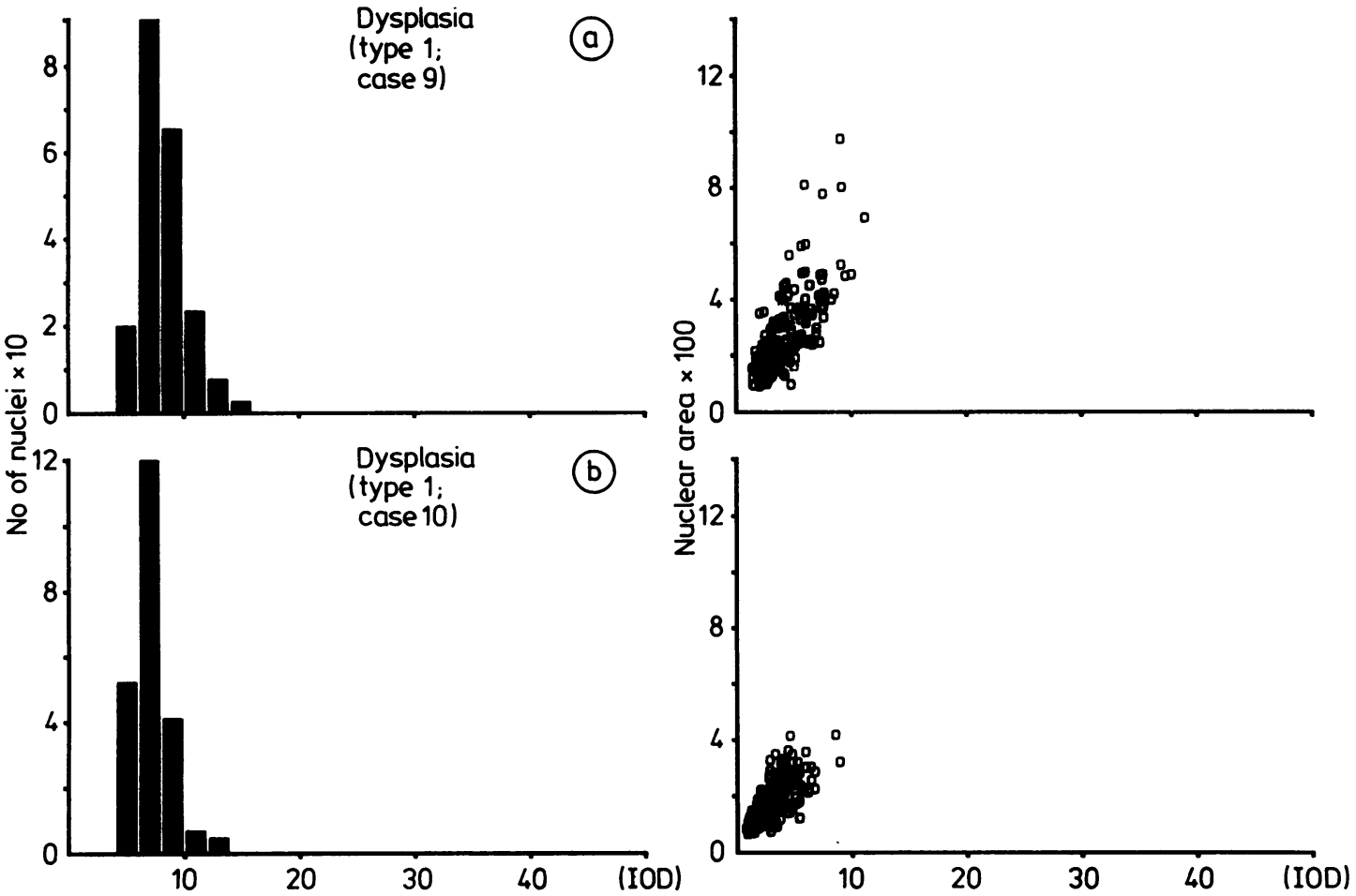

Fig 10 Histograms and scatterplots for two cases of dysplasia associated with adenoma demonstrating typical diploid profile.

found to have simple type 1 profiles, consistent with a normal ploidy composition (fig 10).

\section{Discussion}

The risk of malignancy occurring in an adenomatous polyp is known to be small ${ }^{1}$ and the proportion of dysplasias with ploidy abnormality would be correspondingly low. It is not surprising, therefore, that in this study no ploidy abnormalities were found in the 10 adenomas unassociated with carcinoma. The results indicate, however, that in those adenomatous polyps in which associated carcinoma shows abnormalities of distribution of nuclear ploidy and size, focal regions which by histological definition show evidence of dysplasia but which fall short of the atypia defined as carcinoma, show similar nuclear characteristics. The implication is that the property of invasiveness may be acquired in association with nuclear abnormalities.

Because the present image analysis techniques permit precise correlation with conventional histological assessment, specific focal abnormalities can be investigated. The technique has an additional advan- tage: specimens, which are too small for disaggregation methods, can be analysed. Although the shortcomings of using sections for quantitative nuclear DNA analysis are recognised, ${ }^{9}{ }^{10}$ the present study has shown that interpretation by histogram typing is feasible. Image analysis also provides nuclear size as well as integrated density measurements, which assists in detecting abnormality where the ploidy composition is inconclusive.

Previous studies of ploidy profile in adenomatous polyps may well be marred by a combination of sampling errors. Cell disaggregation randomises the nuclei for analysis and effectively dilutes the abnormal epithelial component with not only other epithelial cells but also with connective tissue elements. Even if a selective surface scraping technique is used, ${ }^{3}$ there is probably still an appreciable amount of unwanted stromal component. Furthermore, the development of carcinoma is often focally distributed in an adenoma and may not be of sufficient volume in proportion to the whole tissue for detection of ploidy abnormality, if cell disaggregation techniques are used. If the focus is centrally located it may not be sampled at all by surface scraping. 
The similarity between nuclear abnormalities in dysplasia and carcinoma in the same lesion, and their absence in the majority of adenomas ${ }^{38}$ supports the hypothesis that dysplasia progresses to carcinoma. This is consistent with the view that the adenomatous polyp to cancer sequence is a multiple stepwise progression determined by disordered cell proliferation indigenous to the adenoma and enhanced by factors in the lumen. ${ }^{11}$ Because these factors combine to produce adenomatous polyps and ensure that adenoma progresses to carcinoma and because colonic carcinoma is so common in Western style societies, it is imperative in terms of cost that some specific feature or features of adenomas be used to select out the patients in need of the closest clinical surveillance, that is, those at greatest risk of developing future adenomatous polyps and carcinoma. The video image analysis techniques used in this study lend themselves to a routine procedure on tissue sections as a convenient adjunct to conventional assessment and they provide a much more accurate index of abnormality than the usual subjective gradings of dysplasia. The method seems to facilitate the selection of adenomatous polyps with the greatest malignant potential.

\section{References}

1 Morson BC. The evolution of colorectal carcinoma. Clin Radiol 1984;35:425-31.
2 Konishi F, Morson BC. Pathology of colorectal adenomas: a colonoscopic survey. J Clin Pathol 1982;35:830-41.

3 van den Ingh HF, Griffioen G, Cornelisse CJ. Flow cytometric detection of aneuploidy in colorectal adenomas. Cancer Res 1985; 45:3392-7.

4 Fand SB. Environmental conditions for optimal Feulgen hydrolysis. In: Weid GL, Bahr GF, eds. Introduction to quantitative cytochemistry II. New York: Academic Press, 1970.

5 Allison DC, Ridolpho PF, Rasch EM, Rasch RW, Johnson TS. Increased accuracy of absorption cytophotometric values by control of stain intensity. J Histochem Cytochem 1981;29: 1219-28.

6 Haggitt CR, Glotzback RE, Soffer EE, Wruble LD. Prognostic factors in colorectal carcinomas arsing in adenomas: implications for lesions removed by endoscopic polypectomy. Gastroenterology 1985;89:328-36.

7 Jarvis LR. A microcomputer system for video image analysis and diagnostic microdensitometry. Anal Quant Cytol Histol 1986;8:201-9.

8 Whitehead F, Jarvis LR, Skinner JM, Whitehead R. Ploidy studies in adenomatous polyps of colon. J Clin Pathol 1985;38:1261-4.

$9 \mathrm{Fu}$ YS, Hall TL. DNA ploidy measurements in tissue sections. Anal Quant Cytol Histol 1985;7:90-5.

10 Nishimiya K, Auer G, Erhardt K, Wiman LG, Kato H, Hayata $Y$. Nuclear DNA in histologic sections from squamous-cell carcinoma and adenocarcinoma of the lung. Anal Quant Cytol Histol 1985;7:153-8.

11 Deschner EE. Adenomas: preneoplastic events, growth and development in man and experimental systems. Pathol Annu 1983;18:205-19.

Requests for reprints to: Professor $\mathbf{R}$ Whitehead, Department of Pathology, Flinders Medical Centre, Bedford Park, South Australia, 5042. 